\title{
Hydrogen emission in enclosed volume (tunnel for mobility)
}

\author{
Farhad Farajimoghadam ${ }^{1,}{ }^{*}$, Matteo Testi $^{1}$, and Luigi Crema ${ }^{1}$ \\ ${ }^{1}$ Sustainable energy group, Fondazione Bruno Kessler, Via Sommarive, 18, 38123, Povo (TN), Italy.
}

\begin{abstract}
Fuel cell vehicles and trains (FCVs) are seen as a viable alternative to fossil fuel-powered vehicles, with the potential to help the automotive and transport industry grow sustainably. Because of their zero emissions, great efficiency, and diverse hydrogen sources, they are an ideal solution to climate change and the global energy issue. In this study, the simulation of releasing hydrogen from a moving vehicle inside a tunnel has been done. For this purpose, two scenarios have been considered. In the first one, it assumed that hydrogen propagates inside a tunnel without ignition and in the second approach, hydrogen released considered to be combusted. The effect of this combustion on the tunnel and train wall has been investigated. For this goal, two different mass flow rates of hydrogen were considered and results were compared together. Moreover, pressure contours have been shown to represent the overpressure phenomenon and it is resulted that in the area of hydrogen dispersion, there will be high pressure.
\end{abstract}

\section{Introduction}

As hydrogen fuel cell vehicle technology advances, it is evident that this mode of transportation will become increasingly widespread in the future. The influence of HFCVs on various road infrastructure must be studied in order to achieve that goal. Tunnels are a major concern because hydrogen might be released and then confined, potentially posing a serious threat[1].

Governments and major vehicle companies have placed a high priority on research and development of FCVrelated technology in recent years. Furthermore, hydrogen seems to be the most acceptable answer for overcoming the current energy and environmental crises from the standpoint of clean energy generation. It may be extracted using solar, wind, or other sustainable energy sources and is commonly available in the form of water. As a result, hydrogen energy and hydrogen FCVs are optimal. This remark ignores the dangers that arise during the manufacturing, storage, transit, and usage processes. Although hydrogen is less likely to create a fire or explosion in an open or well-ventilated room (owing to its ease of diffusion and low density), it can pose a safety danger if it piles up in a confined or poorly ventilated space. While hydrogen is released from the valve, the hydrogen concentration rises for as long as the hydrogen is released. When hydrogen is no longer released, diffusion causes the hydrogen-air mixture to slowly drop. The hydrogen will become homogeneously mixed with the air in the confined space after a long period (far longer than the duration of the discharge). But all this scenario happens if there is not an ignition. When a leak takes place in a hydrogen carrying vehicle, a flammable hydrogen-air mixture might build up within the tunnel, posing a safety threat. Because if the concentration of hydrogen will be more than $4 \%$, the combustion probability of hydrogen-air mixture is so high[2].

When releasing hydrogen, release rate (expressed in $\mathrm{g} / \mathrm{s}$ or $\mathrm{kg} / \mathrm{s}$ ) has a decisive impact on the way in which hydrogen-rich layers form below the ceiling or roof of a space during the release phase[3]. At high release rates, there is usually a lot of turbulence, in which case the hydrogen spreads along the ceiling and walls of the room and descends until the room is homogeneously filled. At low release rates, there is much less turbulence and the hydrogen rises to the ceiling where it forms layers of varying concentrations (stratification). The highest hydrogen concentrations can be found in the layers directly below the ceiling[4]-[6].

Hydrogen dispersion can either be turbulent or laminar in nature. A laminar flow is a steady flow going in one direction, whereas a turbulent flow is much more unpredictable and can go in multiple directions. The higher the pressure of the released gas, the more turbulent the release will be. In the case of a release rate on the order of 1 litre per hour, the flow will be laminar. In the case of more major leaks, the flow will be turbulent[7], [8].

Several research investigations have been conducted both experimentally and numerically that some of them are as follows. Groethe et al. [9] conducted experiments in a $78.5 \mathrm{~m}$ long tunnel with section equal to $3.74 \mathrm{~m} 2$. The tunnel represented approximately $1 / 5$ th scaled real tunnel for vehicles.

In order to validate the dispersion/deflagration modelling, a set of experiments were performed at the SRI Corral Hollow Experiment Site by Houf et al [10]. A set of scaled tunnel tests were performed to approximate the full-scale dimensions of the tunnel from the modelling effort. The hydrogen mass, release rate, initial tank

\footnotetext{
* Corresponding author: ffarajimoghadam@fbk.eu
} 
pressure, and TPRD release diameter were scaled to approximate the modelling parameters.

Other experiments were performed by Kumar et al. [11] to evaluate the influence of congestion and ventilation flow rates on the over-pressure produced from ignition of hydrogen stoichiometric clouds. Quiescent experiments were performed in a sealed enclosure with a stoichiometric hydrogen/air mixture and different congestion volumes/configurations. Also, a set of steadystate experiments were performed with various hydrogen leak rates and ventilation flow rates.

Friedrich et al. [12] and Seike et al. [13] examined deflagration in stratified hydrogen layers to evaluate the potential of selfsustained detonation in flat mixture layers. The hydrogen concentrations used in these experiments ranged between $15 \%$ and $25 \%$ (by volume in air). Hao et al. [14] has been done the experiments related to dispersion of hydrogen from fuel cell vehicles in confined spaces recently.

In terms of numerical investigations, a lot of researchers put efforts as well as $\mathrm{Li}$ et al. [15] that simulated combustion of dispersed hydrogen with CFD code GASFLOW and Choi et al. [16] that did the same for emission of hydrogen from fuel cell vehicles.

Lowesmith et al. [17] worked on the combustion of air hydrogen and methane mixtures with different ratios in the confined spaces and also they investigated the explosion results. Bauwens et al. [18] and Gamezo et al. [19] did some similar studies to define the results regrading the concentration of hydrogen inside confined places.

Computational fluid dynamics is utilized in this study to look into transient and steady-state hydrogen release, dispersion, and explosion in a tunnel for fuel cell moving vehicles.

\section{Modelling of the tunnel}

In this study, computational fluid dynamics has been used to evaluate releasing hydrogen from a moving vehicle like train inside a tunnel. The main objective was obtaining tunnel and train wall temperature considering two cases. These two modes of simulation were consisted of performing calculation with and without burning of hydrogen. The main idea behind this assumption was based on the fact that hydrogen is a very flammable gas with a very little activation energy. Therefore, both cases considering only releases of hydrogen without any ignition and with the spontaneous ignition and combustion were simulated.

\subsection{Geometry}

The geometry information for the tunnel and its crosssection area are given in Fig. 1 and Fig. 2.

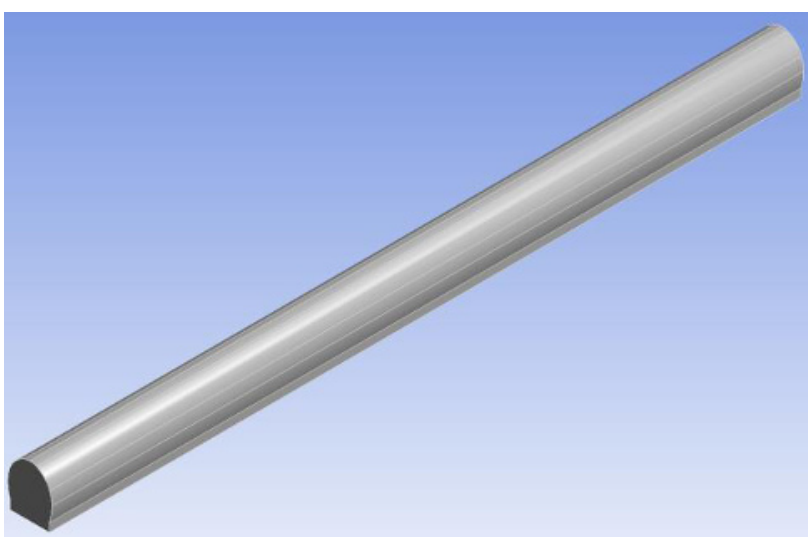

Fig. 1 Tunnel Geometry

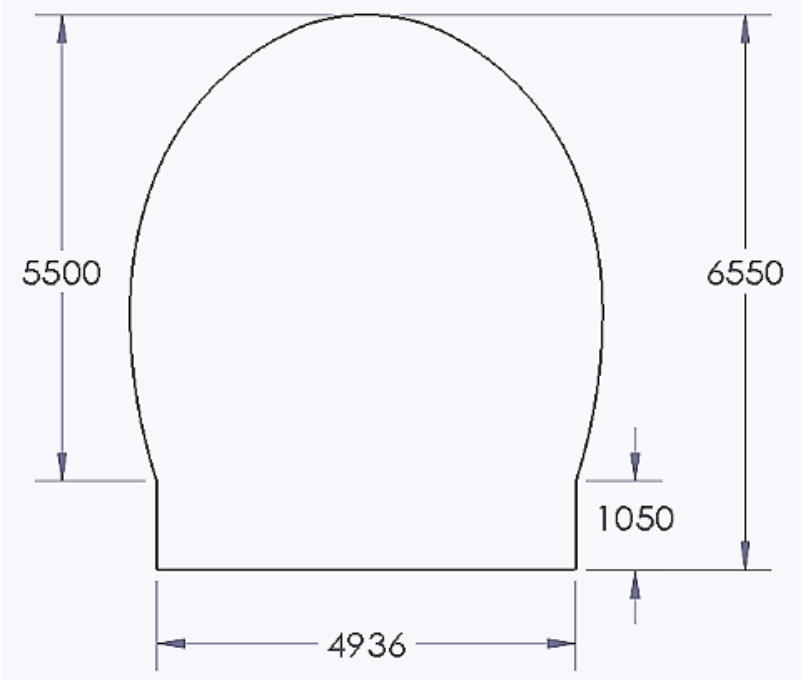

Fig. 2 Cross-section of the tunnel (Dimensions are in millimeters)

Total train length considering the space between wagons is about 100 meters and for this reason a tunnel with the length of more than 100 meters has been chosen to perform all the simulation.

The train composed of several wagons and the middle wagon is the place which hydrogen tanks are and the release of hydrogen happens in the middle of its ceiling.

\subsection{Mesh}

After creating fluid volume from the geometry of tunnel and train, meshing has been done using unstructured meshing technique. For investigating mesh independency, three different mesh from coarse grid to fine have been considered and by comparing the results, it was decided to use the medium mesh with about 7.5 million total nodes. In the Fig. 3, a cross section of mesh is shown: 


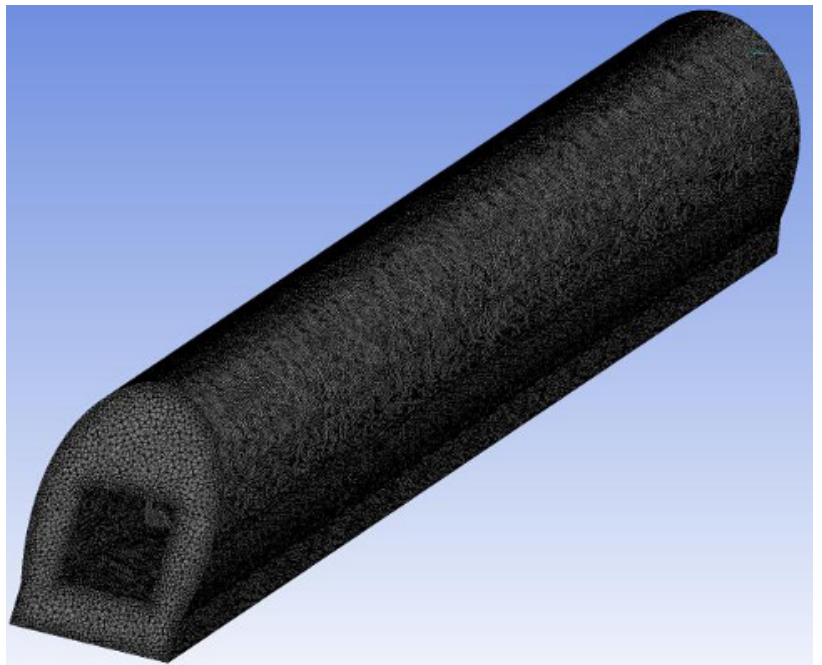

Fig. 3 Cross-section of fluid volume mesh

\subsection{Boundary conditions and assumptions}

A study of hypothetical hydrogen vehicle fire situations in tunnels was undertaken to determine the maximum amount of hydrogen that might be burned in a short period of time.

This research has been done using computational fluid dynamics which is on the basis of finite volume method. First of all, domain is discretized into a finite set of control volumes, after that, general conservation (transport) equations for mass, momentum, energy, species, etc. are solved on this set of control volumes solved on this set of control volumes. Partial differential equations are discretized into a system of algebraic equations and all algebraic equations are then solved numerically to render the solution field.

In this study, it is assumed that train is moving inside the tunnel with the velocity of $80 \mathrm{Km} / \mathrm{hr}$. and hydrogen is released while it is moving. For this reason, for the simulation train was considered stable and instead the flow inside the tunnel is moving with the same velocity. As a result, there is an inlet boundary in one side of tunnel which is considered to be velocity inlet and on the opposite side there should be outlet with the ambient pressure.

For modeling hydrogen, there is another inlet on the train ceiling. Two different amounts of hydrogen were simulated with and without ignition. These two numbers are related to the amount of hydrogen inside the tanks and should be chosen based on this decision that how much hydrogen must be burned or released two alleviate the pressure of the tanks.

Hydrogen release pipe has the diameter of 12 millimeter based on the chosen thermal pressure relief device (TPRD) and it is exactly in the middle of the small coach. This whole idea of releasing hydrogen is due to high pressure of tanks inside the train which can be around 350 bar and if it exceeds from this number, it is so dangerous.

Temperature of air outside tunnel is equal to $300 \mathrm{~K}$ which is ambient temperature and convection heat transfer between burned hydrogen and the outside air was considered.

After burning, fluid is turbulent and for modeling turbulence K-e realizable has been used together with scalable wall functions[20]. The simulation tool extrapolates value of convection heat transfer coefficient based on the various temperatures.

Mass flow rate of hydrogen is constant and two scenarios were evaluated: 1) $140 \mathrm{~g} / \mathrm{s}$ of hydrogen release and 2) 720 $\mathrm{g} / \mathrm{s}$ of hydrogen. Contours are shown and explained in the next section.

\section{Results and discussion}

Release of hydrogen is considered in two ways in this study, first without ignition and second with ignition.

\subsection{Hydrogen release without ignition}

In this section, the released hydrogen will not be burned and a transient simulation has been done in order to know the concentration of hydrogen inside tunnel. Because if the concentration of hydrogen goes over $4 \%$ it is very dangerous and flammable. In this regard, a transient study was carried out and results were extrapolated to know the total time after the first second of hydrogen dispersion to understand the exact of time of reaching this concentration.

Mass flow rate of hydrogen in this section was $140 \mathrm{~g} / \mathrm{s}$ and molar concentration contours has been shown in respect to time in the Fig. 4

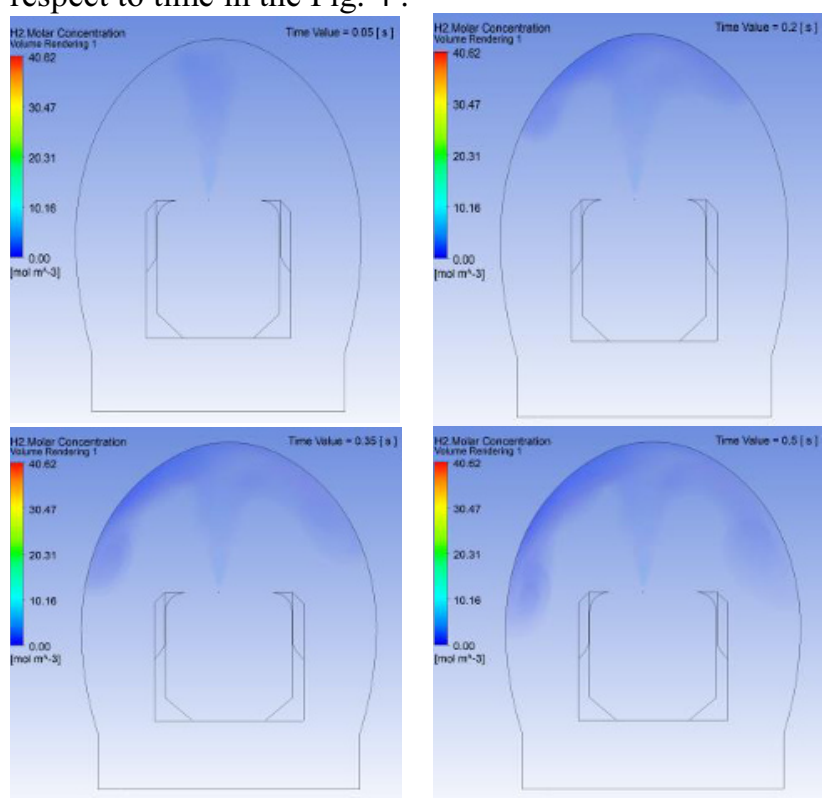

Fig. 4 Hydrogen molar concentration in $\mathrm{mol} / \mathrm{m} 3$ in different times

From data above a relation has been derived that shows the relation of hydrogen concentration inside tunnel in respect to time which is shown in the Fig. 5.

As it is obvious, Concentration of hydrogen increases gradually and after one minute it goes higher than $4 \%$, exactly $4.2 \%$. A linear relationship was found between time and concentration since the mass flow rate of hydrogen dispersioni s constant during the time.

As a matter of fact, it seems that after one minute or 60 seconds the mixture of hydrogen and air in the tunnel has become extremely flammable. It is better to note that the concentration of hydrogen is based on total volume of tunnel and it is average. As a result, in some parts of tunnel ceiling it can be higher. 


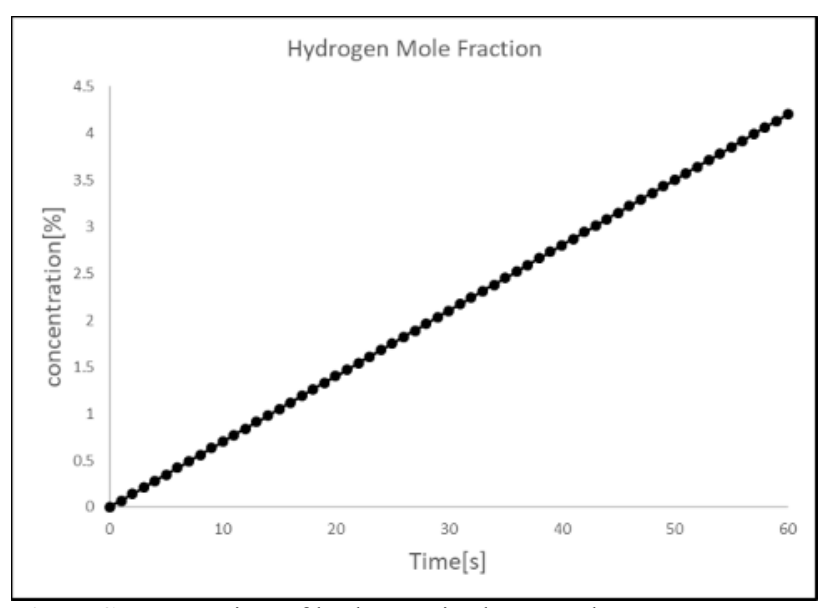

Fig. 5 Concentration of hydrogen in the tunnel

\subsection{Hydrogen release with ignition}

In this part, the effect of burning hydrogen after dispersion will be shown. For burning the stoichiometric ratio of hydrogen and air was considered and all the simulations have been carried out in steady state mode.

The main goals were calculating adiabatic hydrogen flame temperature and evaluation train and tunnel wall temperature. For this reason, two cases were examined. Hydrogen mass flow rate for these cases was $140 \mathrm{~g} / \mathrm{s}$ and $720 \mathrm{~g} / \mathrm{s}$ respectively.

Contour of adiabatic flame temperature for the first case is shown in the Fig. 6.

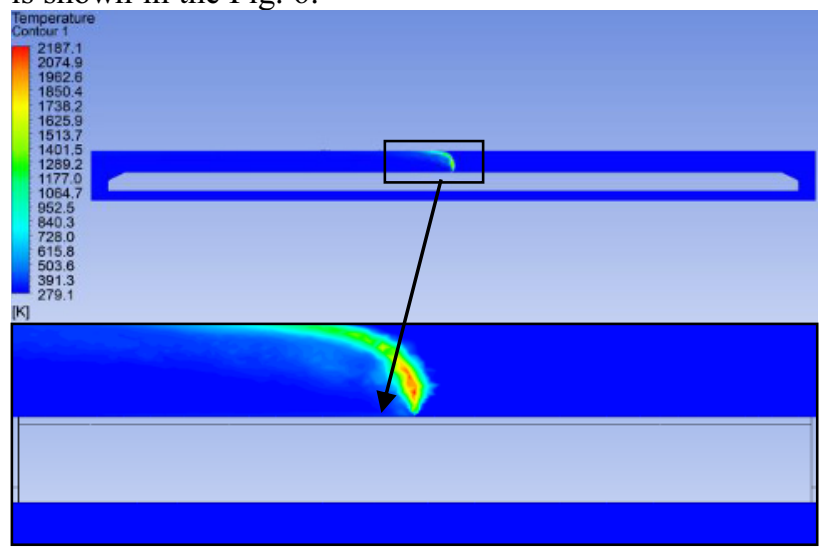

Fig. 6 Hydrogen flame temperature in the tunnel centerline (for $140 \mathrm{~g} / \mathrm{s}$ of hydrogen)

Maximum flame temperature is around $2200 \mathrm{~K}$ which is close to the hydrogen adiabatic flame temperature, furthermore, the effect of burning hydrogen on the tunnel ceiling is exhibited in the Fig. 7.

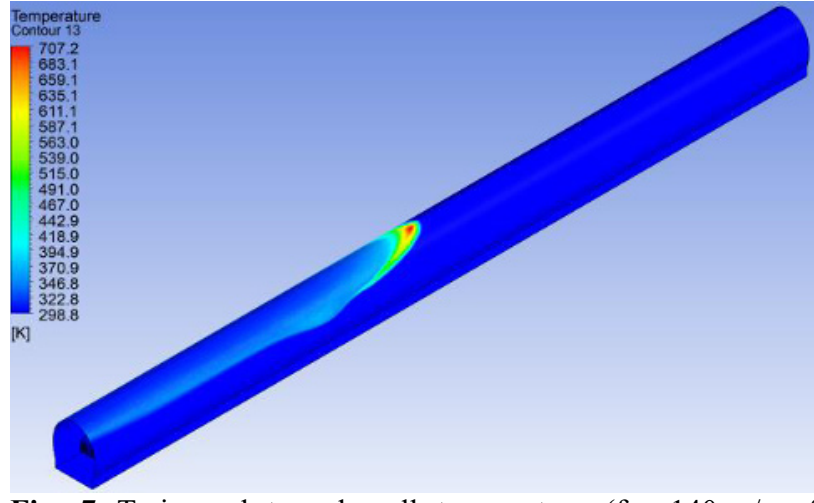

Fig. 7 Train and tunnel wall temperature (for $140 \mathrm{~g} / \mathrm{s}$ of hydrogen)

As it can be seen, the steady state effect of burning $140 \mathrm{~g} / \mathrm{s}$ hydrogen is clear with a hot spot area just above the release point and maximum temperature on the tunnel wall is equal to $707 \mathrm{~K}$ which represents 434 degrees centigrade.

In the figure Fig. 8, contours of water and oxygen mass fraction can be seen. As it is expected, in the area of combustion, oxygen fully consumed and water was produced as a product of combustion.

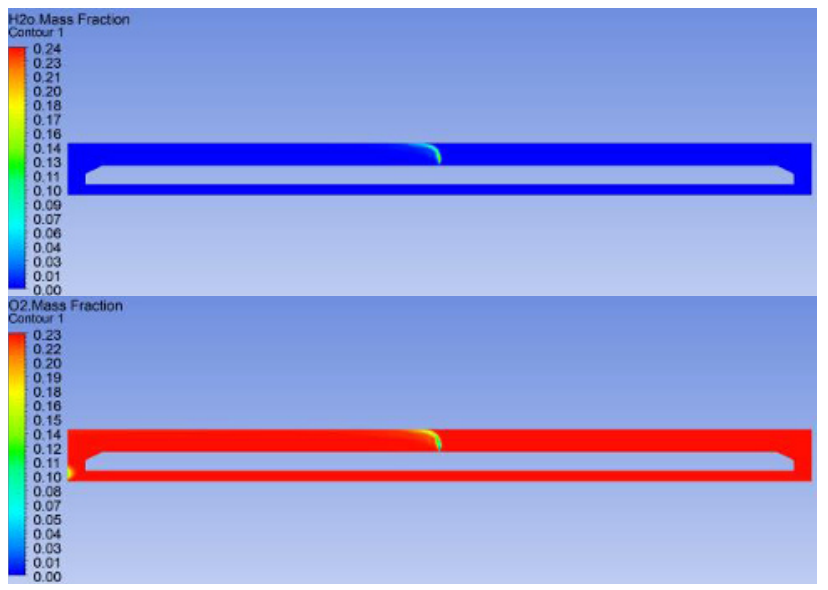

Fig. 8 Water and oxygen mass fraction in tunnel centerline

In the figure Fig. 9, volume rendering temperature inside the tunnel is exhibited which can be a representative of the temperature in every point of tunnel.

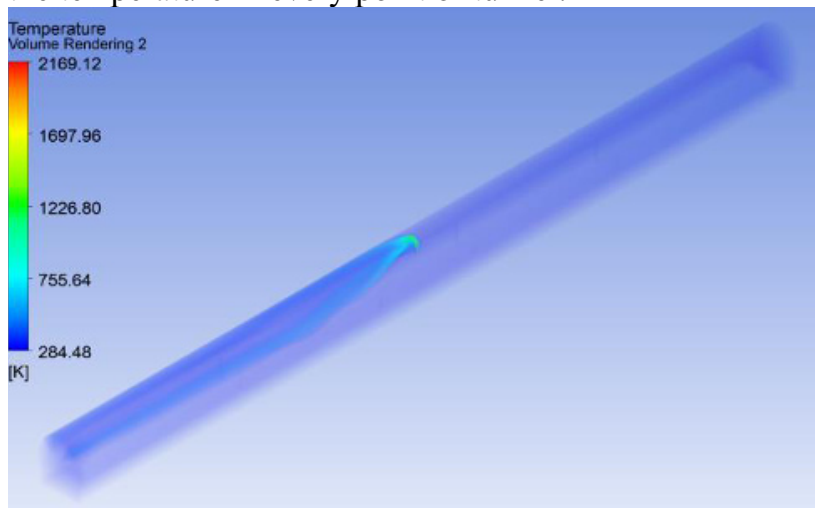

Fig. 9 Volume rendering temperature of train and tunnel (for $140 \mathrm{~g} / \mathrm{s}$ of hydrogen) 
For digging in about the pressure in the tunnel, three different section were chosen along the tunnel including the central section. In the figure Error! Reference source not found., overpressure can be observed in middle section due to the release of hydrogen from a very little $12 \mathrm{~mm}$ diameter pipe. In the other sections, contours of pressure show the pressure difference with the reference.

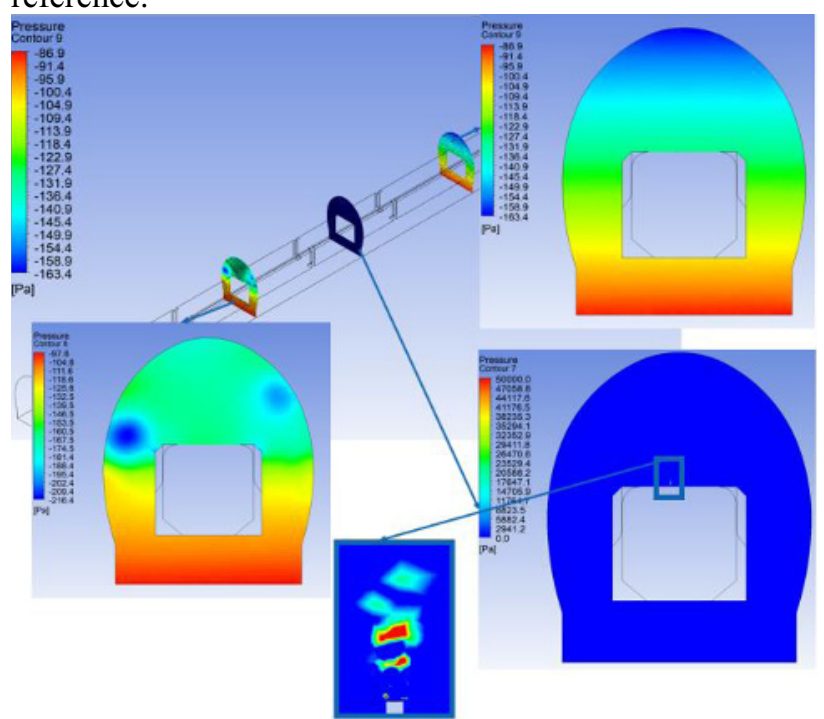

Fig. 10 Pressure contours in three different sections (for $140 \mathrm{~g} / \mathrm{s}$ of hydrogen)

In the second case, everything is the same unlss the mass flow rate of hydrogen which is $720 \mathrm{~g} / \mathrm{s}$.

Contour of tempreature in the tunnel centerline is shown in the Fig. 11, the only difference is the total area which had been covered by the heat and as it is obvious a very bigger area has high temperature. Additionally, maximum flame temperature is almost constant and is around 2200 $\mathrm{K}$ like before, because it should not be dependent to the amount of burned hydrogen.

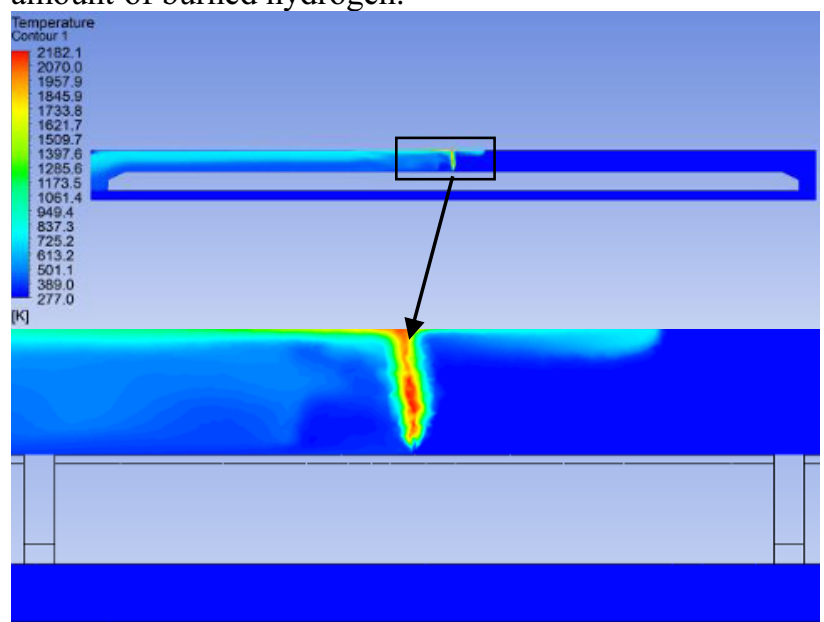

Fig. 11 Hydrogen flame temperature in the tunnel centerline (for $720 \mathrm{~g} / \mathrm{s}$ of hydrogen)

In the , temperature contour is exhibited on the train and tunnel wall. In this regarad, with increasing the amount of burned hydrogen, maximum flame temperaure does not change but maximum tunnel wall temperature shows a significant change which is a consequence of more heat produced by hydrogen burning.

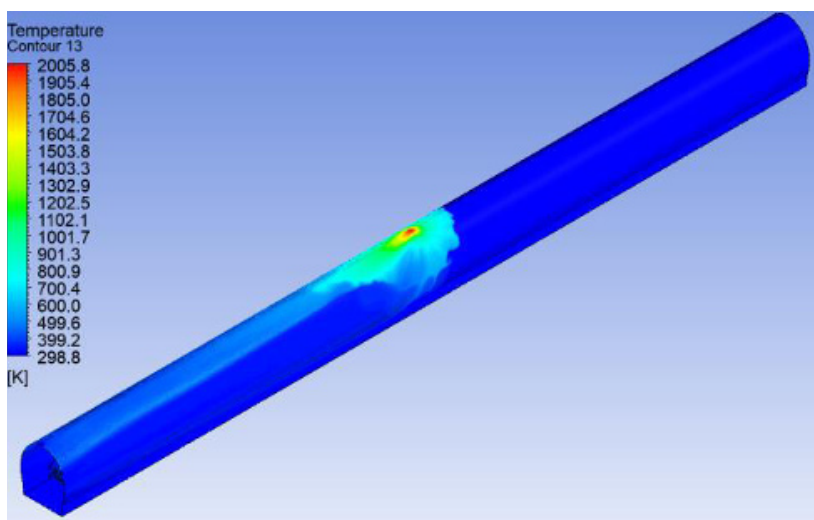

Fig. 12 Train and tunnel wall temperature (for $720 \mathrm{~g} / \mathrm{s}$ of hydrogen)

Water and oxygen mass fraction do not represent a huge deviation with previouse results due to the similarity of the sotoichiometric ratio and they will not be shown again. On the other side, it is interesting to depict the volume rendering temperature in the new case while more volume of fluid inside the tunnel have been affected by the combustion.

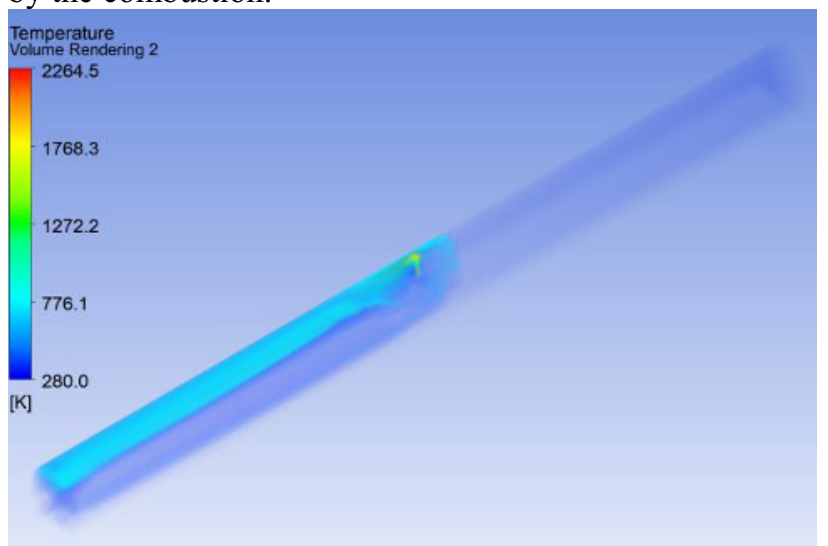

Fig. 13 Volume rendering temperature of train and tunnel (for $720 \mathrm{~g} / \mathrm{s}$ of hydrogen)

Regarding pressure contours, the same ones have been depicted for the second case and what is significant is that in general, pressure is higher which easily can be explained. The diameter of release point is constant but amount of hydrogen flow rate is more. Therefore, overpressure happens. They were exhibited in Fig. 14. 


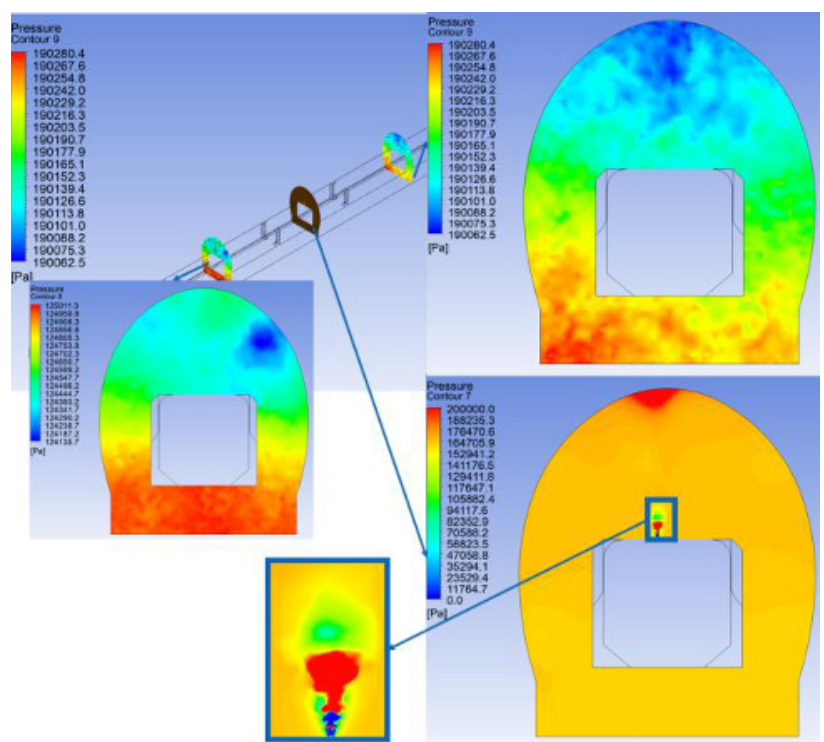

Fig. 14 Pressure contours in three different sections (for $720 \mathrm{~g} / \mathrm{s}$ of hydrogen)

In the pressure contours, negative pressure shown in the contour is due to the relative pressure in comparison with working pressure. Also, the blue holes in pressure contours are related to the velocity of combustion products which are obvious only in the plane after releasing hydrogen.

\section{Conclusions}

In this research, computational fluid dynamics is used to investigate transient and steady-state hydrogen release, dispersion, and explosion in a tunnel for fuel cell moving vehicles. The following are some of the inferences that can be drawn:

-Releasing hydrogen into air without ignition, apparently does not have a big effect on growing train wall temperature, but after one minute it can cause an autoignition of hydrogen because the concentration of hydrogen inside tunnel goes above $4 \%$ after 1 minute and it could be dangerous.

-Increasing mass flow rate of hydrogen, increases maximum train wall temperature and approximately does not have a big effect on flame temperature because the hydrogen adiabatic flame temperature depends on stoichiometric ratio of fuel and air.

-It is assumed that releasing of hydrogen starts when the train is in the middle of tunnel but in reality, it can happen in any point of tunnel and has the same effect of increasing temperature of train and tunnel.

-After passing a few seconds, increasing time of simulation does not change maximum temperature.

-Increasing the velocity and pressure of flow inside the tunnel is due to the very small release diameter of hydrogen and from the simulation it is concluded that in other parts of tunnel high pressure and velocity does not exist as it was shown in the pressure and velocity contours.

\section{References}

[1] KIWA, "Future-proof gas distribution networks," 2018, Accessed: Sep. 02, 2021. [Online].

Available: www.kiwatechnology.nl.

[11] S. Kumar, S. D. Miles, P. Adams, A. Kotchourko, D. Hedley, and P. Middha, "Hytunnel project to investigate the use of Hydrogen Vehicles in Road Tunnels," International Conference on Hydrogen Safety (ICHS3), 2009.

https://businessdocbox.com/Green_Solutions/815 70426-Hytunnel-project-to-investigate-the-useof-hydrogen-vehicles-in-road-tunnels.html (accessed Sep. 02, 2021).

[12] K. S. and G. A. Friedrich, J. Grune, T. Jordan, A. Kotchourko, N. K. M. Kotchourko and Stern, "EXPERIMENTAL STUDY OF HYDROGENAIR DEFLAGRATIONS IN FLAT LAYER," 2007.

[13] M. H. and H. T. M. Seike, Y. Ejiri, N. Kawabata, ""Fire experiments of carrier loaded FCV in fullscale model tunnel - Estimation of heat release rate and smoke generation rate," 2014.

[14] D. Hao, X. Wang, Y. Zhang, R. Wang, G. Chen, 
and J. Li, "Experimental Study on Hydrogen Leakage and Emission of Fuel Cell Vehicles in Confined Spaces," Automot. Innov. 2020 32, vol. 3, no. 2, pp. 111-122, May 2020, doi: 10.1007/S42154-020-00096-Z.

[15] Y. Li et al., "Numerical analysis of hydrogen release, dispersion and combustion in a tunnel with fuel cell vehicles using all-speed CFD code GASFLOW-MPI," Int. J. Hydrogen Energy, vol. 46, no. 23, pp. 12474-12486, Mar. 2021, doi: 10.1016/J.IJHYDENE.2020.09.063.

[16] J. Choi, S. Kang, N. Hur, E. D. Lee, and K. B. Lee, "A NUMERICAL SIMULATION OF HYDROGEN DIFFUSION FOR THE HYDROGEN LEAKAGE FROM A FUEL CELL VEHICLE IN AN UNDERGROUND PARKING GARAGE."

[17] B. J. Lowesmith, C. Mumby, G. Hankinson, and J. S. Puttock, "Vented confined explosions involving methane/hydrogen mixtures," Int. J. Hydrogen Energy, vol. 36, no. 3, pp. 2337-2343, Feb. 2011, doi:

10.1016/J.IJHYDENE.2010.02.084.
[18] C. R. Bauwens, J. Chao, and S. B. Dorofeev, "EFFECT OF HYDROGEN CONCENTRATION ON VENTED EXPLOSION OVERPRESSURES FROM LEAN HYDROGEN-AIR DEFLAGRATIONS."

[19] V. N. Gamezo, T. Ogawa, and E. S. Oran, "Numerical simulations of flame propagation and DDT in obstructed channels filled with hydrogenair mixture," doi: 10.1016/j.proci.2006.07.220.

[20] T. J. Bardina, J.E., Huang, P.G., Coakley, "Turbulence Modeling Validation, Testing, and Development," NASA Technical Memorandum 110446., 1997.

https://www.researchgate.net/publication/242962 13_Turbulence_Modeling_Validation_Testing_a nd_- Development (accessed Sep. 07, 2021). 